\title{
Improvements in Intractable Lumbar and Lower- Extremity Symptoms after Systemic Administration of Tocilizumab, an Anti-interleukin-6 Receptor Antibody
}

\author{
Takeshi Sainoh $^{1}$, Sumihisa Orita ${ }^{2,3}$, Masayuki Miyagi ${ }^{4}$, Miyako Suzuki-Narita ${ }^{2,5}$, Yoshihiro Sakuma ${ }^{6}$, \\ Yasuhiro Oikawa ${ }^{7}$, Go Kubota ${ }^{8}$, Jun Sato ${ }^{2}$, Yasuhiro Shiga ${ }^{2}$, Kazuki Fujimoto ${ }^{9}$, Yawara Eguchi ${ }^{2}$, \\ Masao Koda ${ }^{10}$, Yasuchika Aoki ${ }^{11}$, Tsutomu Akazawa ${ }^{12}$, Takeo Furuya ${ }^{2}$, Junichi Nakamura ${ }^{2}$, \\ Hiroshi Takahashi ${ }^{10}$, Satoshi Maki ${ }^{2}$, Masahiro Inoue ${ }^{11}$, Hideyuki Kinoshita ${ }^{13}$, Masaki Norimoto ${ }^{14}$, \\ Takashi Sato ${ }^{2}$, Masashi Sato ${ }^{2}$, Masahiro Suzuki ${ }^{2}$, Keigo Enomoto ${ }^{2}$, Hiromitsu Takaoka ${ }^{2}$, \\ Norichika Mizuki ${ }^{2}$, Takashi Hozumi ${ }^{2}$, Ryuto Tsuchiya ${ }^{2}$, Geundong Kim², Takuma Otagiri ${ }^{2}$, \\ Tomohito Mukaihata ${ }^{2}$, Takahisa Hishiya ${ }^{2}$, Seiji Ohtori ${ }^{2}$, Kazuhide Inage ${ }^{2}$ \\ ${ }^{1}$ Department of Orthopaedic Surgery, Sainou Hospital, Toyama, Japan \\ ${ }^{2}$ Department of Orthopaedic Surgery, Graduate School of Medicine, Chiba University, Chiba, Japan \\ ${ }^{3}$ Center for Frontier Medical Engineering, Chiba University, Chiba, Japan \\ ${ }^{4}$ Department of Orthopaedic Surgery, School of Medicine, Kitasato University, Sagamihara, Japan \\ ${ }^{5}$ Department of Environmental Medicine, Graduate School of Medicine, Chiba University, Chiba, Japan \\ ${ }^{6}$ Department of Orthopaedic Surgery, Kanazawa Hospital, Yokohama, Japan \\ ${ }^{7}$ Department of Orthopaedic Surgery, Chiba Children's Hospital, Chiba, Japan \\ ${ }^{8}$ Department of Orthopaedic Surgery, Chiba Prefectural Sawara Hospital, Katori, Japan \\ ${ }^{9}$ Department of Orthopaedic Surgery, Kohnodai Hospital, Ichikawa, Japan \\ ${ }^{10}$ Department of Orthopaedic Surgery, Faculty of Medicine, University of Tsukuba, Tsukuba, Japan \\ ${ }^{11}$ Department of Orthopaedic Surgery, Eastern Chiba Medical Center, Togane, Japan \\ ${ }^{12}$ Department of Orthopaedic Surgery, St. Marianna University School of Medicine, Kawasaki, Japan \\ ${ }^{13}$ Department of Orthopaedic Surgery, Chiba Cancer Center, Chiba, Japan \\ ${ }^{14}$ Department of Orthopaedic Surgery, Toho University Sakura Medical Center, Sakura, Japan
}

Study Design: Prospective cohort study (open-label, single-arm, and non-blinded).

Purpose: This study aims to determine the effects of systemic administration of tocilizumab, an anti-interleukin-6 (IL-6) receptor antibody on refractory low back pain and leg symptoms.

Overview of Literature: IL-6 overexpression is associated with neuropathic pain pathogenesis, which is potentially followed by chronic low back pain, including leg pain and numbness. This finding suggest that inhibition of IL-6 at the site of pain or in the transmission pathway could provide novel therapeutic targets for chronic low back pain.

Methods: This prospective, single-arm study included 11 patients (eight men; mean age, 62.7 years) with $\geq 3$-months' chronic pain history due to lumbar disease. Subcutaneous TCZ injections were administered twice, at a 2-week interval. We evaluated low back pain, leg pain, and leg numbness using numeric rating scales and the Oswestry Disability Index (ODI; baseline and 6 months postinjection); serum IL-6 and tumor necrosis factor- $\alpha$ levels (baseline and 1 month postinjection); and clinical adverse events.

Received Jun 5, 2020; Revised Oct 2, 2020; Accepted Oct 25, 2020

Corresponding author: Kazuhide Inage

Department of Orthopedic Surgery, Graduate School of Medicine, Chiba University, 1-8-1 Inohana Chuo-ku, Chiba 260-8670, Japan

Tel: +81-43-226-2117, Fax: +81-43-226-2116, E-mail: kazuhideinage@chiba-u.jp 
Results: Intractable symptoms reduced after TCZ administration. Low back pain improved for 6 months. Improvements in leg pain and numbness peaked at 4 and 1 month, respectively. Improvements in ODI were significant at 1 month and peaked at 4 months. Serum IL-6 was increased at 1 month. IL-6 responders (i.e., patients with IL-6 increases $>10 \mathrm{pg} / \mathrm{mL}$ ) showed particularly significant improvements in leg pain at 2 weeks, 1 month, and 2 months compared with nonresponders. We observed no apparent adverse events.

Conclusions: Systemic TCZ administration improved symptoms effectively for 6 months, with peak improvements at 1-4 months and no adverse events. Changing serum IL-6 levels correlated with leg pain improvements; further studies are warranted to elucidate the mechanistic connections between lumbar disorders and inflammatory cytokines.

Keywords: Interleukin-6; Chronic pain; Low back pain; Analgesic; Tocilizumab

\section{Introduction}

Low back pain (LBP) is a major issue in orthopedic pathology that requires the use of significant medical resources [1] and has a lifetime incidence of approximately $80 \%$ [2]. The primary cause of LBP is degeneration or rupture of lumbar structural tissues, such as the intervertebral disc (IVD), facet joint, or muscle structure [3,4]. Patients with LBP are typically treated with medication, injections, and rehabilitation therapy. However, LBP symptoms generally do not improve, and chronic LBP develops because of the insufficient effectiveness of current treatments [5]. LBP results from the overexpression of pivotal inflammatory cytokines, such as interleukin-6 (IL-6) and tumor necrosis factor- $\alpha$ (TNF- $\alpha$ ), in degenerated lumbar structures [6]. Furthermore, the overexpression of IL-6 can contribute to the pathogenesis of neuropathic pain [7], which can be followed by chronic LBP, leg pain, and numbness. Thus, IL-6 inhibition at the site of pain or in the transmission pathway could provide novel therapeutic targets for LBP. Tocilizumab (TCZ), which is a humanized anti-IL-6 receptor monoclonal antibody that blocks IL- 6 from binding to its receptor [8], has shown excellent therapeutic efficacy and safety in patients with rheumatoid arthritis $[9,10]$. We previously reported the results of epidural TCZ administration for the treatment of radicular leg pain with lumbar spinal stenosis; however, the observed analgesic effect lasted for only 4 weeks. Moreover, there is no evidence on the efficacy of systemic TCZ administration for the management of pain in lumbar diseases. We hypothesized that systemic TCZ administration might confer a long-term analgesic effect on the pain associated with lumbar diseases. In this study, we evaluated the efficacy and safety of systemic TCZ administration in patients with chronic LBP and related symptoms such as leg pain and numbness.

\section{Materials and Methods}

We performed an assessment of our hypothesis that systemic TCZ administration might be efficacious and safe to treat chronic LBP, leg pain, and numbness in patients with lumbar diseases who show resistance to conservative therapies. Our study was prospective, open-label, singlearm, and nonblinded. Ethical approval was provided by the institutional review board of the University of Chiba, Japan (IRB approval no., G26026), and all participants provided written informed consent.

Approximately 3,000 patients with chief complaints of lumbar and lower-extremity symptoms visited our outpatient clinic over 1 year; we enrolled 14 of these patients (nine men and five women; mean age, 63.0 years) who showed resistance to conservative therapies (medication, rehabilitation therapy, or injection) for longer than 3 months. The inclusion criteria were a baseline numeric rating scale (NRS) pain score $>4$ ( 0 , no pain; 10 , worst pain) and an Oswestry Disability Index (ODI) $>30 \%$. Individuals with spinal or spinal cord tumors, infection, trauma, or psychiatric disorders were excluded. The enrolled patients were diagnosed with lumbar spinal stenosis, degenerative spondylolisthesis, or lumbar disc diseases based on their clinical symptoms and radiological findings. Five patients had previously undergone lumbar spinal surgery, and two patients had undergone multiple back operations. Blood examinations were performed on all patients before drug administration. Any patients who had serious medical disorders, such as a high inflammatory response; untreated diabetes; infectious diseases; or 
heart, lung, liver, or renal disorders were excluded. Baseline ODI and NRS scores for LBP, leg pain, and leg numbness were recorded for each patient and were based on the results of a questionnaire. Subsequently, systemic TCZ administration by subcutaneous injection (162 mg) was performed twice after rheumatoid arthritis therapy, with a 2 -week interval between injections [11]. Serum IL-6 and TNF- $\alpha$ levels were measured before and at 1 month after TCZ administration.

We evaluated the following data: (1) temporal changes in self-reported pain evaluation using the NRS score (LBP, leg pain, and numbness before TCZ administration; at 1, 7, and 14 days after TCZ administration; and at 1, 2, 4, and 6 months after TCZ administration); (2) temporal changes in self-reported daily activity evaluation based on the ODI (before TCZ administration and at 1,2, 4, and 6 months after); (3) changes in serum IL- 6 and TNF- $\alpha$ levels (before TCZ administration versus 1 month after); and (4) any significant variation in the amount of cytokine changes. Furthermore, patients were divided into responder and nonresponder subgroups based on the rate of serum IL-6 change (described later), and the change in NRS scores was compared between these two subgroups. Finally, we recorded and evaluated any clinical adverse events.

Paired comparisons of NRS and ODI scores with $\geq 3$ time points were analyzed by Friedman's test. Betweengroup comparisons were conducted employing the Wil- coxon $t$-test. The Mann-Whitney $U$-test was used for comparisons between the responder and nonresponder groups and comparisons of each serum cytokine level. A $p$-value $<0.05$ was considered statistically significant for all analyses.

\section{Results}

One patient refused the second TCZ administration because the visit was inconvenient. Two patients elected to undergo surgical treatment within 2 months after TCZ administration. The remaining 11 patients (eight men, three women; mean age, $62.7 \pm 13.4$ years) were followed for 6 months. Table 1 shows the baseline patient characteristics. LBP, leg pain, and leg numbness were reduced after TCZ administration (Fig. 1). LBP was significantly reduced at 2 weeks after TCZ administration, and the pain reduction continued for 6 months. Leg pain was significantly reduced at 1 week after TCZ administration, with pain reduction peaking at 4 months after TCZ administration, and tending to slightly worsen at 6 months. Leg numbness was significantly reduced 1 day after TCZ administration, and the effect peaked at 4 months, which was similar to the effect of TCZ administration on leg pain. The patients' ODI was significantly improved at 1 month after TCZ administration; the ODI improvement tended to continue until 4 months and then slightly worsened at 6 months

Table 1. Pain score and serum cytokine level before drug administration

\begin{tabular}{|c|c|c|c|c|c|c|c|c|}
\hline \multirow{2}{*}{ Case no. } & \multirow{2}{*}{ Age (yr) } & \multirow{2}{*}{ Diagnosis } & \multicolumn{3}{|c|}{ NRS } & \multirow{2}{*}{ ODI } & \multicolumn{2}{|c|}{ Serum cytokines } \\
\hline & & & LBP & Leg pain & Leg numbness & & IL-6 (pg/mL) & TNF- $\boldsymbol{\alpha}(\mathrm{pg} / \mathrm{mL})$ \\
\hline 1 & 71 & LSS & 7 & 7 & 0 & 67.8 & 1.3 & 1.2 \\
\hline 2 & 63 & LSS & 10 & 10 & 10 & 70.0 & 7.9 & 38.0 \\
\hline 3 & 83 & DDD & 8 & 7 & 7 & 56.0 & 3.9 & 11.5 \\
\hline 4 & 47 & DDD & 9 & 0 & 0 & 40.0 & 1.1 & 1.4 \\
\hline 5 & 49 & DDD & 4 & 0 & 0 & 28.0 & 0.9 & 1.1 \\
\hline 6 & 52 & LDH & 7 & 6 & 7 & 40.0 & 11.7 & 31.0 \\
\hline 7 & 74 & LSS & 5 & 5 & 5 & 37.8 & 3.0 & 12.4 \\
\hline 8 & 73 & LSS & 8 & 8 & 2 & 54.0 & 2.4 & 2.8 \\
\hline 9 & 63 & LSS & 4 & 3 & 8 & 18.0 & 1.8 & 0.9 \\
\hline 10 & 73 & FS & 3 & 8 & 5 & 42.2 & 9.1 & 24.3 \\
\hline 11 & 42 & $\mathrm{DDD}$ & 10 & 3 & 5 & 32.0 & 1.3 & 1.4 \\
\hline Mean $\pm S D$ & $62.7 \pm 13.4$ & & $6.8 \pm 2.5$ & $5.2 \pm 2.3$ & $4.5 \pm 3.0$ & $43.3 \pm 4.8$ & $4.0 \pm 1.1$ & $11.5 \pm 3.7$ \\
\hline
\end{tabular}

NRS, numeric rating scale; LBP, low back pain; ODI, Oswestry Disability Index; IL-6, interleukin-6; TNF- $\alpha$, tumor necrosis factor- $\alpha$; LSS, lumbar spinal stenosis; DDD, degenerative disc disease; LDH, lumbar disc herniation; FS, foraminal stenosis; SD, standard deviation. 


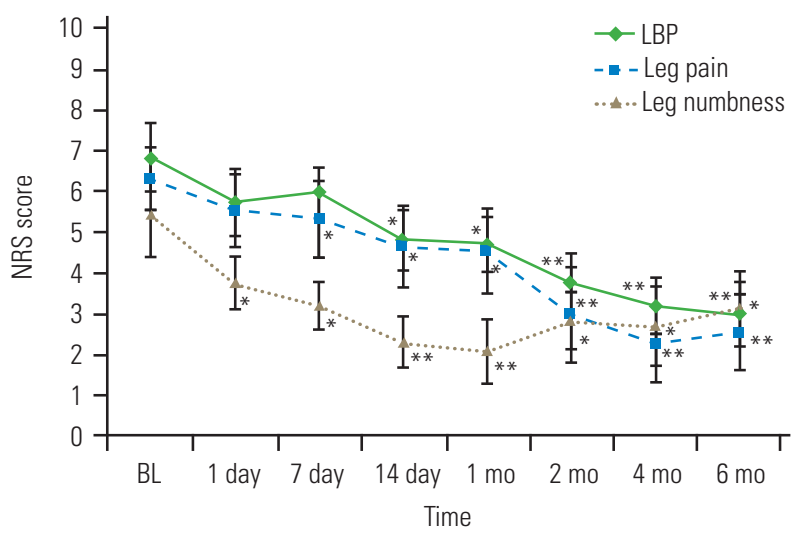

Fig. 1. All numeric rating scale (NRS) scores showed significant improvement after systemic tocilizumab administration. Low back pain (LBP) improved for up to 6 months. The peak of the improvement in leg pain was observed at 4 months, whereas the peak of the improvement in leg numbness was observed at 1 month. Error bars represent the standard error of the mean. BL, baseline. ${ }^{*} p<0.05$ and ${ }^{* *} p<0.01$ compared with BL.

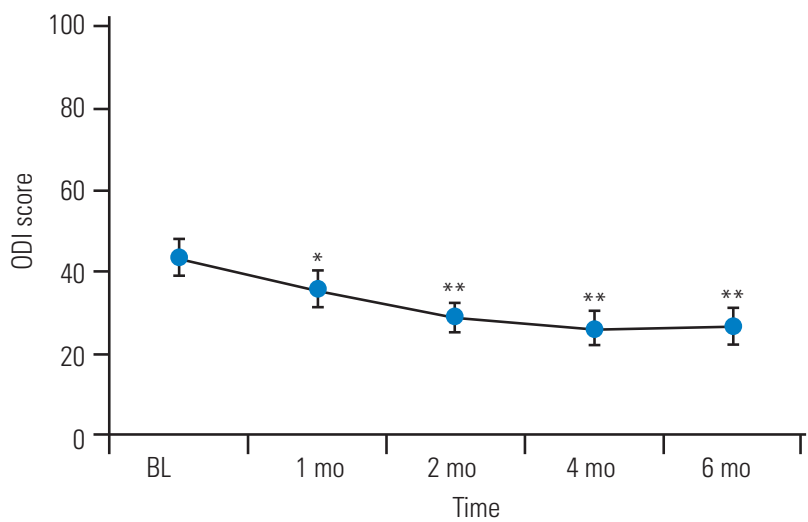

Fig. 2. Oswestry Disability Index (ODI) was significantly improved at 1 month compared with the baseline value. The effect on ODI tended to continue for 4 months, and was slightly restored at 6 months. Error bars represent the standard error of the mean. $\mathrm{BL}$, baseline. ${ }^{*} p<0.05$ and ${ }^{* *} p<0.01$ compared with $\mathrm{BL}$.

(Fig. 2).

The patients' serum IL-6 levels were increased at 1 month after systemic TCZ administration; however, their serum TNF- $\alpha$ levels decreased after systemic TCZ administration (Fig. 3). The increase in the patients' serum IL-6 levels was statistically significant ( $p=0.0026$ ), which suggested that patients with a more drastic change in serum IL-6 level might experience more effective pain relief. Therefore, we assigned the patients to responder and nonresponder groups based on the change in their serum cytokine levels. Patients were assigned to the responder group if they showed serum IL- 6 changes of $>10 \mathrm{pg} / \mathrm{mL}$; otherwise, they were assigned to the nonresponder group (Fig. 4). We compared the responder and nonresponder
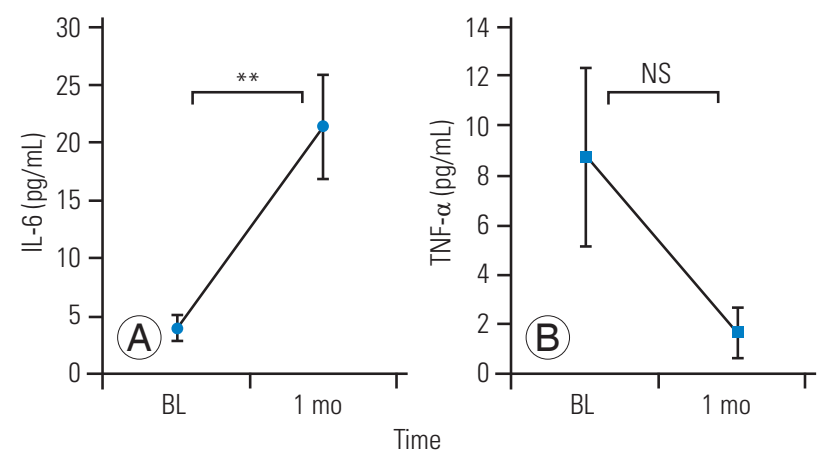

Fig. 3. (A, B) The mean serum interleukin-6 (IL-6) and tumor necrosis factor- $\alpha$ (TNF- $\alpha$ ) levels had increased and decreased, respectively, at 1 month after systemic tocilizumab administration. Error bars represent the standard deviation. BL, baseline; NS, not significant. ${ }^{* *} p<0.01$ compared with BL.

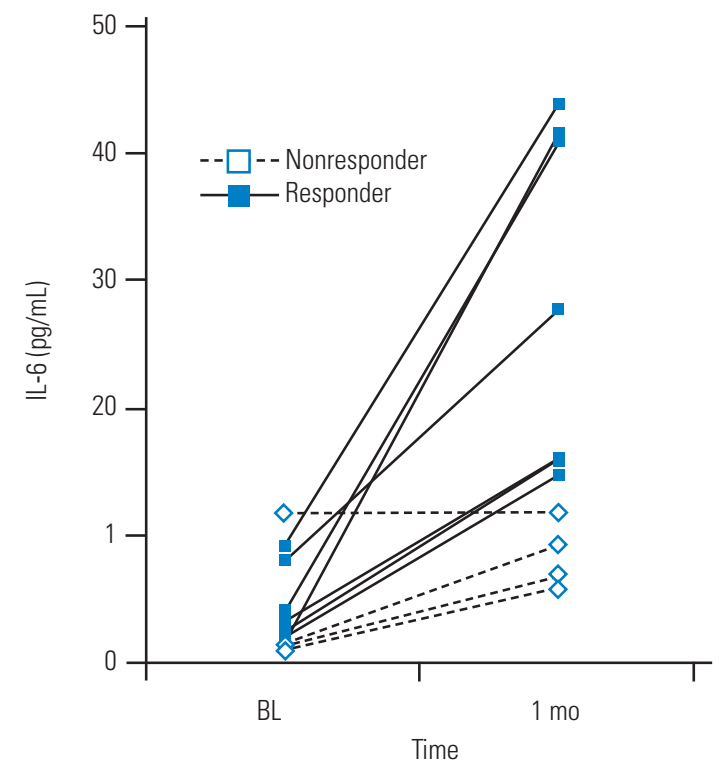

Fig. 4. Based on the changes in their serum interleukin-6 (IL-6) levels, the patients were divided into responder (increase of $>10 \mathrm{pg} / \mathrm{mL}$ ) and nonresponder groups ( $<10 \mathrm{pg} / \mathrm{mL})$. BL, baseline.

groups' NRS scores collected at preadministration; at 1, 7, and 14 days after TCZ administration; and at 1,2, 4, and 6 months after TCZ administration. Table 2 shows the differences in background data (age, sex, baseline severity) between the responder and nonresponder groups. The responder group had a significantly greater improvement in NRS score for leg pain at 14 days, 1 month, and 2 months post-administration of TCZ than the nonresponder group. However, the NRS scores for LBP and leg numbness showed no significant differences between the groups at any time point (Fig. 5).

We observed no apparent adverse effects associated with TCZ administration, such as increased pain or numbness, 
Table 2. Background data (nonresponder versus responder)

\begin{tabular}{lccc} 
Variable & Nonresponder $(\mathrm{n}=7)$ & Responder $(\mathrm{n}=4)$ & $p$-value \\
Age (yr) & $61.9 \pm 12.7$ & $64.3 \pm 16.4$ & $<0.05$ \\
Sex & & & $<0.05$ \\
$\quad$ Male & 5 & 3 & \\
$\quad$ Female & 2 & 1 & \\
Diagnosis (case) & & & $>0.05$ \\
$\quad$ LSS & 6 & - & \\
\multicolumn{1}{c}{ DDD } & 1 & 3 & \\
\hline FS & 1 & - & \\
\hline LDH & - & 1 & \\
LBP (NRS) & $6.4 \pm 2.5$ & $7.5 \pm 2.6$ & $<0.05$ \\
\hline Leg pain (NRS) & $6.9 \pm 2.1$ & $4.5 \pm 2.1$ & $<0.05$ \\
Leg numbness (NRS) & $5.3 \pm 3.5$ & $6.0 \pm 1.4$ & $<0.05$ \\
\hline ODI & $48.1 \pm 18.2$ & $35.0 \pm 6.0$ & $<0.05$ \\
\hline
\end{tabular}

Values are presented as mean \pm standard deviation or number.

LSS, lumbar spinal stenosis; DDD, degenerative disc disease; FS, foraminal stenosis; LDH, lumbar disc herniation; LBP, low back pain; NRS, numeric rating scale; ODI, Oswestry Disability Index. symptoms of infection (including fever), or increased preexisting disease during the study period.

\section{Discussion}

Systemic TCZ administration by subcutaneous injection reduced the symptoms of chronic LBP, leg pain, and leg numbness without adverse effects. The reduction in LBP in our patients remained throughout the 6-month followup period. However, the reductions in leg pain and numbness reached their maximum at 4 months and 1 month, respectively, after which the pain tended to increase gradually. Similarly, the improvement in ODI peaked at 4 months. Among the serum cytokine levels analyzed, the IL-6 level increased significantly. The increase in serum IL-6 levels correlated with the reduction in leg pain at 14 days, 1 month, and 2 months after TCZ administration.

IL-6 is expressed in pain transmission pathways, which include sites peripheral to the central nervous system,

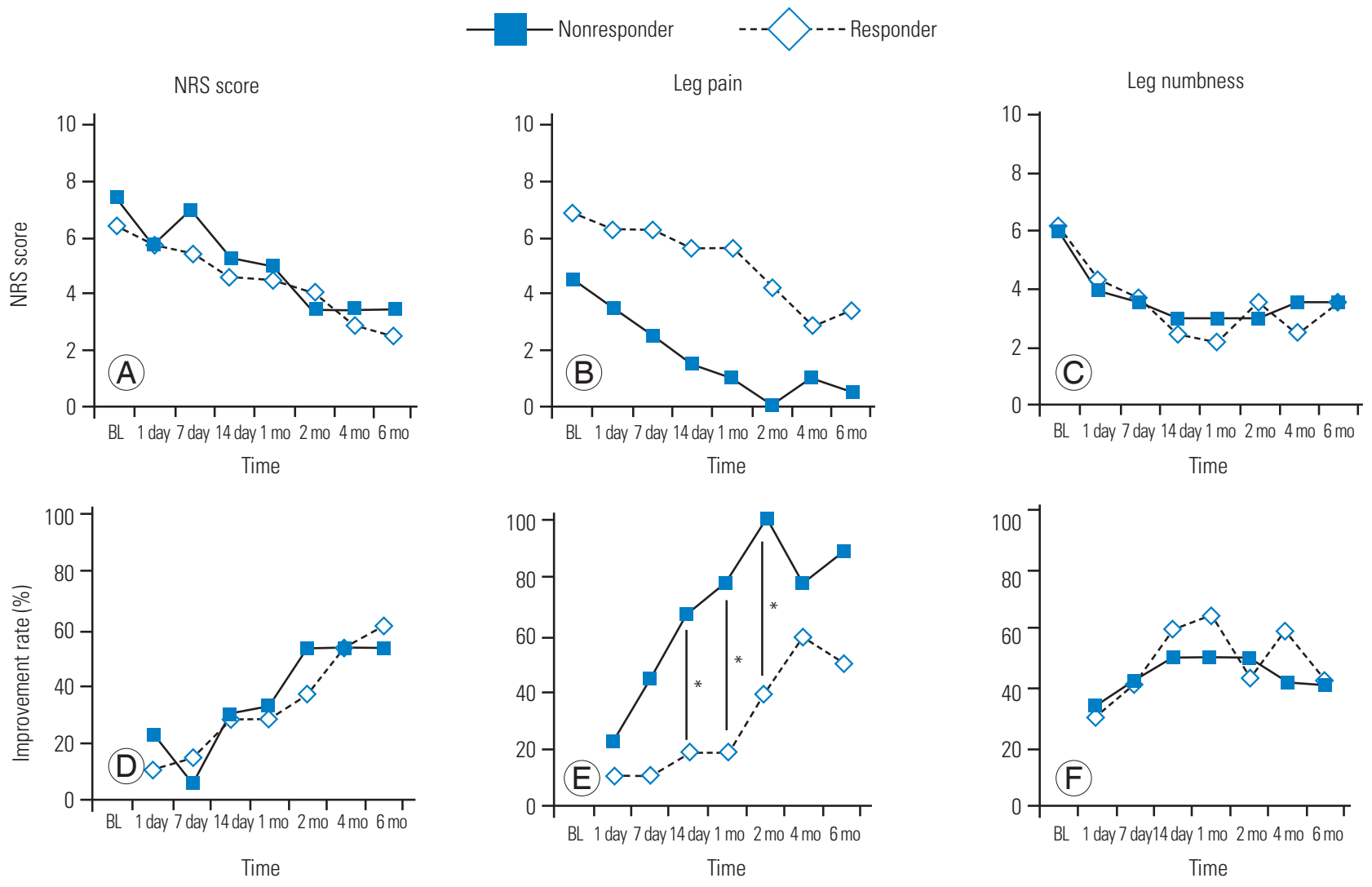

Fig. 5. (A-F) The interleukin-6 (IL-6) responder group had significantly greater rates of leg pain improvement at 2 weeks, 1 month, and 2 months post-administration $\left({ }^{*} p<0.05\right)$ compared with the IL-6 nonresponder group, although the improvement rates for low back pain (LBP) and leg numbness did not differ significantly between the groups at any of the investigated time points. NRS, numeric rating scale; BL, baseline. 
and may contribute to pain $[12,13]$. Animal models have shown increased IL-6 expression at the site of pain in IVD injury [6,14], peripheral nerve injury [15], human degenerated IVD [16], the facet joint [17], and cerebrospinal fluid [18]. In addition, increased IL-6 expression in the pain transmission pathway of spinal cord injury and sciatic nerve injury has been reported in animal models $[19,20]$. Moreover, a mouse spinal cord injury model has shown that intrathecal administration of an anti-IL-6 receptor mouse monoclonal antibody (MR16-1) improved pain-related behavior [21].

We previously reported that an epidural TCZ administration to the spinal nerve reduced radicular leg pain; however, the pain relief continued for only 1 month [22]. In addition, we reported that an intradiscal TCZ injection provided an analgesic effect for only 1 month in patients with discogenic LBP [23]. These studies showed that an inhibition of IL-6 limited to the painful local lumbar structure did not elicit a clinically useful pain relief effect. Furthermore, block therapy was performed by perineural puncture, and its invasiveness cannot be ignored. Therefore, we hypothesized that systemic TCZ administration could effectively reduce pain by affecting both the peripheral and central nervous systems. Moreover, systemic TCZ administration by subcutaneous injection is less invasive than other methods of administration.

TCZ is a humanized IL-6 receptor monoclonal antibody that is widely used in the treatment of rheumatoid arthritis, juvenile idiopathic arthritis, and adult-onset Still's disease. These diseases cause a systemic inflammatory condition and degeneration of the joints and surrounding tissue. TCZ suppresses the disease state in these disorders by inhibiting the overexpression of IL- 6 that binds to the receptor. In our study, the mean serum IL-6 level in our patients increased after systemic TCZ administration. Serum IL- 6 binds to the soluble IL- 6 receptor or membrane IL-6 receptor, which mediates IL-6 signaling into the cell [24]. TCZ might have prevented this signaling pathway and caused transient upregulation of the serum IL-6 level via a feedback mechanism. We speculate that effective inhibition of the IL- 6 signaling pathway resulted in the increased serum IL-6 level observed in our patients.

The change in serum IL-6 levels correlated with the reduction in leg pain. IL-6 genetic variations are associated with degenerative disc disease (DDD), including sciatica [2]. DDD and its associated lower-extremity symptoms are characterized by tissue destruction, inflammation, and pain, all of which can be related to excessive IL- 6 function. Thus, an IL-6 haplotype is associated with DDD and sciatica [25]. In the current study, the reduction in leg pain of the responder group was better than that of the nonresponder group. The increase in serum IL-6 (nonbinding IL-6) levels might have been the result of significant binding inhibition of IL-6 and IL-6R. Thus, IL-6 signaling (excessive and abnormal function related to intractable pain) was satisfactorily suppressed in the responder group, which might have led to their lower-extremity symptom improvements.

We observed no obvious adverse events related to systemic TCZ administration in this study. However, a previous study had reported infections in $41.6 \%$ of the patients in the TCZ-subcutaneous (SC) group and in $45.1 \%$ of those in the TCZ-intravenous (IV) group [11]. Nasopharyngitis was the most common event, with a prevalence of $17.9 \%$ in the TCZ-SC group and $20.8 \%$ in the TCZ-IV group. Serious infections occurred in $1.2 \%$ of the patients in the TCZ-SC group and in $2.9 \%$ of those in the TCZ-IV group [11]. Our study enrolled patients with chronic LBP and excluded those with significant comorbidities. In addition, the patients received subcutaneous administrations of TCZ only twice. These factors could have contributed to the lack of adverse events.

Systemic TCZ administration was performed by subcutaneous injection, which does not have the significant drug delivery complications of other methods, such as intravenous administration [11]. The advantages of subcutaneous injection are less patient burden, easy selfadministration, and no preparation. Repeated TCZ administration represents a change in the traditional medical treatment for LBP, which is typically provided via nonsteroidal anti-inflammatory drugs, acetaminophen, or other approaches.

The present study has some limitations. First, this study was not a controlled or randomized study. Future studies should include a control group treated with normal saline via subcutaneous injection and should be performed in a blinded manner. The present study was a preliminary investigation; further randomized, controlled trials are essential. Second, this study was not compared with other types of treatment, such as NSAIDs, pregabalin, tramadol, or intrathecal injection of TCZ. Third, the number of patients was small, and they had various diagnoses. In a future study, patients with chronic LBP and lower-extremity symptoms should be evaluated for each disorder. 
Fourth, we performed subcutaneous TCZ injection only twice, even though its effects continued for 4-6 months. In cases of rheumatoid arthritis, TCZ administration is performed continuously based on inflammatory findings and clinical observation of the pain sites. The efficacy of continuous and repeated administration should be examined accordingly. Finally, changes in cytokine levels in the central nervous system, which includes the brain, spinal cord, and blood, were not fully evaluated in the present study. It is unclear how TCZ acts or leads to pain relief in the body (especially the pain pathway). An animal model should be developed, and evaluations of inflammatory cytokines should be performed. Further studies should be performed to elucidate the mechanistic connection between lumbar disorders and inflammatory cytokines and to establish effective and safe anti-cytokine therapy.

\section{Conclusions}

We investigated the efficacy and safety of systemic TCZ administration in patients with lumbar and lower-extremity symptoms. No apparent adverse effects were observed in our patients, and pain, numbness, and activities of daily living improved for 4-6 months. The changes in serum IL-6 levels correlated with leg pain reduction.

\section{Conflict of Interest}

No potential conflict of interest relevant to this article was reported.

\section{Author Contributions}

Takeshi Sainoh wrote and prepared the manuscript, and all of the authors participated in the study design. All authors have read, reviewed, and approved the article.

\section{References}

1. Dagenais S, Caro J, Haldeman S. A systematic review of low back pain cost of illness studies in the United States and internationally. Spine J 2008;8:8-20.

2. Airaksinen O, Brox JI, Cedraschi C, et al. Chapter 4: European guidelines for the management of chronic nonspecific low back pain. Eur Spine J 2006;15(Suppl 2):S192-300.

3. Takahashi K, Aoki Y, Ohtori S. Resolving discogenic pain. Eur Spine J 2008;17(Suppl 4):428-31.

4. Schwarzer AC, Aprill CN, Derby R, Fortin J, Kine G, Bogduk N. The prevalence and clinical features of internal disc disruption in patients with chronic low back pain. Spine (Phila Pa 1976) 1995;20:1878-83.

5. Andersson GB. Epidemiological features of chronic low-back pain. Lancet 1999;354:581-5.

6. Miyagi M, Ishikawa T, Kamoda $\mathrm{H}$, et al. ISSLS prize winner: disc dynamic compression in rats produces long-lasting increases in inflammatory mediators in discs and induces long-lasting nerve injury and regeneration of the afferent fibers innervating discs: a pathomechanism for chronic discogenic low back pain. Spine (Phila Pa 1976) 2012;37:1810-8.

7. Mukaino M, Nakamura M, Yamada O, et al. Anti-IL6-receptor antibody promotes repair of spinal cord injury by inducing microglia-dominant inflammation. Exp Neurol 2010;224:403-14.

8. Mihara M, Kasutani K, Okazaki M, et al. Tocilizumab inhibits signal transduction mediated by both mIL-6R and sIL-6R, but not by the receptors of other members of IL-6 cytokine family. Int Immunopharmacol 2005;5:1731-40.

9. Nishimoto N, Miyasaka N, Yamamoto K, et al. Study of active controlled tocilizumab monotherapy for rheumatoid arthritis patients with an inadequate response to methotrexate (SATORI): significant reduction in disease activity and serum vascular endothelial growth factor by IL-6 receptor inhibition therapy. Mod Rheumatol 2009;19:12-9.

10. Smolen JS, Beaulieu A, Rubbert-Roth A, et al. Effect of interleukin- 6 receptor inhibition with tocilizumab in patients with rheumatoid arthritis (OPTION study): a double-blind, placebo-controlled, randomised trial. Lancet 2008;371:987-97.

11. Ogata A, Tanimura K, Sugimoto T, et al. Phase III study of the efficacy and safety of subcutaneous versus intravenous tocilizumab monotherapy in patients with rheumatoid arthritis. Arthritis Care Res (Hoboken) 2014;66:344-54.

12. Burke JG, Watson RW, McCormack D, Dowling FE, Walsh MG, Fitzpatrick JM. Intervertebral discs which cause low back pain secrete high levels of proinflammatory mediators. J Bone Joint Surg Br 2002;84:196201.

13. Hurst SM, Wilkinson TS, McLoughlin RM, et al. Il-6 and its soluble receptor orchestrate a temporal switch 
in the pattern of leukocyte recruitment seen during acute inflammation. Immunity 2001;14:705-14.

14. Sainoh T, Orita S, Miyagi M, et al. Interleukin-6 and interleukin- 6 receptor expression, localization, and involvement in pain-sensing neuron activation in a mouse intervertebral disc injury model. J Orthop Res 2015;33:1508-14.

15. Chen ZL, Yu WM, Strickland S. Peripheral regeneration. Annu Rev Neurosci 2007;30:209-33.

16. Lee S, Moon CS, Sul D, et al. Comparison of growth factor and cytokine expression in patients with degenerated disc disease and herniated nucleus pulposus. Clin Biochem 2009;42:1504-11.

17. Igarashi A, Kikuchi S, Konno S, Olmarker K. Inflammatory cytokines released from the facet joint tissue in degenerative lumbar spinal disorders. Spine (Phila Pa 1976) 2004;29:2091-5.

18. Ohtori S, Suzuki M, Koshi T, et al. Proinflammatory cytokines in the cerebrospinal fluid of patients with lumbar radiculopathy. Eur Spine J 2011;20:942-6.

19. Guerrero AR, Uchida K, Nakajima H, et al. Blockade of interleukin-6 signaling inhibits the classic pathway and promotes an alternative pathway of macrophage activation after spinal cord injury in mice. J Neuroinflammation 2012;9:40.
20. Lee KM, Jeon SM, Cho HJ. Interleukin-6 induces microglial CX3CR1 expression in the spinal cord after peripheral nerve injury through the activation of p38 MAPK. Eur J Pain 2010;14:682.

21. Murakami T, Kanchiku T, Suzuki H, et al. Anti-interleukin-6 receptor antibody reduces neuropathic pain following spinal cord injury in mice. Exp Ther Med 2013;6:1194-8.

22. Ohtori S, Miyagi M, Eguchi Y, et al. Efficacy of epidural administration of anti-interleukin-6 receptor antibody onto spinal nerve for treatment of sciatica. Eur Spine J 2012;21:2079-84.

23. Sainoh T, Orita S, Miyagi M, et al. Single intradiscal injection of the interleukin-6 receptor antibody tocilizumab provides short-term relief of discogenic low back pain; prospective comparative cohort study. J Orthop Sci 2016;21:2-6.

24. Sato K, Tsuchiya M, Saldanha J, et al. Reshaping a human antibody to inhibit the interleukin 6-dependent tumor cell growth. Cancer Res 1993;53:851-6.

25. Noponen-Hietala N, Virtanen I, Karttunen R, et al. Genetic variations in IL6 associate with intervertebral disc disease characterized by sciatica. Pain 2005;114:186-94. 